\title{
Quantum criticality of Mott transition in organic materials
}

\author{
Tetsuya Furukawa ${ }^{1 \star}$, Kazuya Miyagawa $^{1}$, Hiromi Taniguchi ${ }^{2}$, Reizo Kato ${ }^{3}$ and Kazushi Kanoda ${ }^{1 \star}$
}

\begin{abstract}
A many-body quantum system on the verge of instability between two competing ground states may exhibit quantumcritical phenomena ${ }^{1,2}$, as has been intensively studied for magnetic systems. The Mott metal-insulator transition ${ }^{3}$, a phenomenon that is central to many investigations of strongly correlated electrons, is also supposed to be quantum critical, although this has so far not been demonstrated experimentally. Here, we report experimental evidence for the quantum-critical nature of the Mott instability, obtained by investigating the electron transport of three organic systems with different ground states under continuously controlled pressure. The resistivity obeys the material-independent quantum-critical scaling relation bifurcating into a Fermi liquid or Mott insulator, irrespective of the ground states. Electrons on the verge of becoming delocalized behave like a strange quantum-critical fluid before becoming a Fermi liquid.
\end{abstract}

Mutually interacting electrons with sufficiently strong Coulomb repulsion $U$ fall into the Mott insulating state when the carrier density corresponds to an electron per site (a half-filled band) ${ }^{3}$. As the bandwidth $W$ is increased by pressure or chemical substitution, the electrons gain kinetic energy and become itinerant at a critical value of $W / U$. The Mott transition, a marked phase transition between a metal and an insulator, is a collective manifestation of imbalance in the particle-wave duality of electrons. As one of the main issues in the quantum physics of condensed matter, the quantumcritical nature of the Mott transition awaits clarification. In contrast to intensive theoretical studies ${ }^{4-6}$, however, this issue has not yet been addressed experimentally because most Mott transitions in real systems have critical points at finite temperatures ${ }^{7-11}$; thus, they are not genuine quantum phase transitions.

In general, quantum criticality is observed at the temperature $T$ sufficiently lower than the competing energy scales underlying the phase transition ${ }^{1,2}$, which are the bandwidth $W$ and on-site Coulomb energy $U$ in the case of the Mott transition. Thus, even if the system's critical point, $T_{c}$, is finite, unlike the genuine quantum phase transition, in the case that $T_{\mathrm{c}}$ is orders of magnitude lower than $W$ and $U$, there is a vast temperature region of $T_{c}<T \ll U, W$, where the system can experience quantum criticality (Fig. 1a). Indeed, using dynamical mean field theory (DMFT), which can properly describe the Mott transition ${ }^{12}$, the authors of refs 4,13 have suggested the scaling of transport for quantum criticality in an intermediate temperature range well above $T_{c}$.

To explore the possible Mott quantum criticality from the experimental side, we performed pressure studies of the electron transport for three different quasi-two-dimensional organic Mott insulators with anisotropic triangular lattices, $\kappa-(\mathrm{ET})_{2} \mathrm{Cu}_{2}(\mathrm{CN})_{3}$, $\kappa-(\mathrm{ET})_{2} \mathrm{Cu}\left[\mathrm{N}(\mathrm{CN})_{2}\right] \mathrm{Cl}$ and $\mathrm{EtMe}_{3} \mathrm{Sb}\left[\mathrm{Pd}(\mathrm{dmit})_{2}\right]_{2}$ (hereafter abbreviated to $\kappa-\mathrm{Cu}_{2}(\mathrm{CN})_{3}, \kappa-\mathrm{Cl}$ and $\mathrm{EtMe}_{3} \mathrm{Sb}$-dmit, respectively), where
ET and dmit represent bis(ethylenedithio)tetrathiafulvalene and 1,3-dithiole-2-thione-4,5-dithiolate, respectively (Fig. 1b,c). In the Mott insulating phases, $\kappa-\mathrm{Cu}_{2}(\mathrm{CN})_{3}$ and $\mathrm{EtMe}_{3} \mathrm{Sb}$-dmit host quantum spin liquids (QSLs), whereas $\kappa-\mathrm{Cl}$ is an antiferromagnet ${ }^{14-18}$ $(\mathrm{AFM})$. In the metallic phases, $\kappa-\mathrm{Cu}_{2}(\mathrm{CN})_{3}$ and $\kappa-\mathrm{Cl}$ are superconducting (SC) at low temperatures, whereas $\mathrm{EtMe}_{3} \mathrm{Sb}$-dmit remains a paramagnetic metal ${ }^{9-11,19-21}(\mathrm{PM})$. Then, the three systems have different types of Mott transition in their ground states, for example, QSL-SC, AFM-SC and QSL-PM transitions (Fig. 1d-f). Clear firstorder Mott transitions are observed in $\kappa-\mathrm{Cu}_{2}(\mathrm{CN})_{3}$ and $\kappa-\mathrm{Cl}$ up to $T_{c}$ values of $20 \mathrm{~K}$ (ref. 22) and $38 \mathrm{~K}$ (ref. 11), respectively, whereas there is no clear first-order nature in the Mott transition in $\mathrm{EtMe}_{3} \mathrm{Sb}$ $\mathrm{dmit}^{21}$; its critical temperature, if any, is well below $30 \mathrm{~K}$. The critical temperatures of the three compounds are two or three orders of magnitude lower than the values of $W, U$, which are several thousand Kelvin or more ${ }^{19}$ (Fig. 1a); the orders-of-magnitude difference between $T_{c}$ and $U, W$ preserves the possibility of quantum criticality in the intermediate temperature region $\left(T_{\mathrm{c}}<T \ll U, W\right)$. We measured resistivity curves $\rho(P, T)$ under continuously controlled He-gas pressure $P$ at various fixed temperatures to cover the metalinsulator crossover region and tested the quantum-critical scaling of the $\rho(P, T)$ data. Figure $1 \mathrm{~d}-\mathrm{f}$ present coloured contour plots of the normalized $\rho(P, T)$ (explained later in detail), which is shown to follow the quantum-critical scaling almost perfectly in the fan-shaped region where the colour changes, as described in the following section.

First, we define metal-insulator crossover pressures at a given temperature, $P_{c}(T)$, as inflection points in the experimental $\log \rho(P, T)$ versus $P$ curve (Supplementary Information). The $P_{c}(T)$ determined at different temperatures forms a bowshaped crossover line, which corresponds to the Widom line of the Mott transition ${ }^{4,13}$. The Widom line divides the insulating $\left(\delta P \equiv P-P_{c}(T)<0\right)$ and metallic sides $(\delta P>0)$, as observed in Fig. 1d-f. Figure 2 shows the normalized resistivity $\tilde{\rho}(\delta P, T) \equiv \rho(\delta P, T) / \rho_{\mathrm{c}}(T)$ of $\kappa-\mathrm{Cu}_{2}(\mathrm{CN})_{3}$ as a function of $\delta P$, where $\rho_{\mathrm{c}}(T) \equiv \rho(\delta P=0, T)$ is the crossover resistivity along the Widom line. It is observed that $\tilde{\rho}(\delta P, T)$ crosses continuously from the insulating state $(\delta P<0)$ to the metallic state $(\delta P>0)$. As a result of the normalization, all curves cross at a single point for which $\delta P=0$ and $\tilde{\rho}=1$, and the slope at the inflection point is steeper at lower temperatures. It is noted that the volume change of the sample, which can be large particularly near the Mott transition, has no practical influence on the $\rho(P, T)$ values, as explained in the Supplementary Information.

For a quantum phase transition ${ }^{1,2}$, as a system approaches a quantum-critical point while remaining at zero temperature, not only the spatial correlation length $\xi$ but also the correlation time $\tau$ diverges as $\xi \propto\left|g-g_{\mathrm{c}}\right|^{-v}$ and $\tau \propto \xi^{z} \propto\left|g-g_{\mathrm{c}}\right|^{-z v}$, where $g$ is the

${ }^{1}$ Department of Applied Physics, University of Tokyo, 7-3-1 Hongo, Bunkyo-ku, Tokyo 113-8656, Japan. ${ }^{2}$ Department of Physics, Saitama University, 255 Shimo-Okubo, Sakura-ku, Saitama-shi, Saitama 338-8570, Japan. ${ }^{3}$ RIKEN, 2-1 Hirosawa, Wako-shi, Saitama 351-0198, Japan.

*e-mail: furukawa@ap.t.u-tokyo.ac.jp; kanoda@ap.t.u-tokyo.ac.jp 

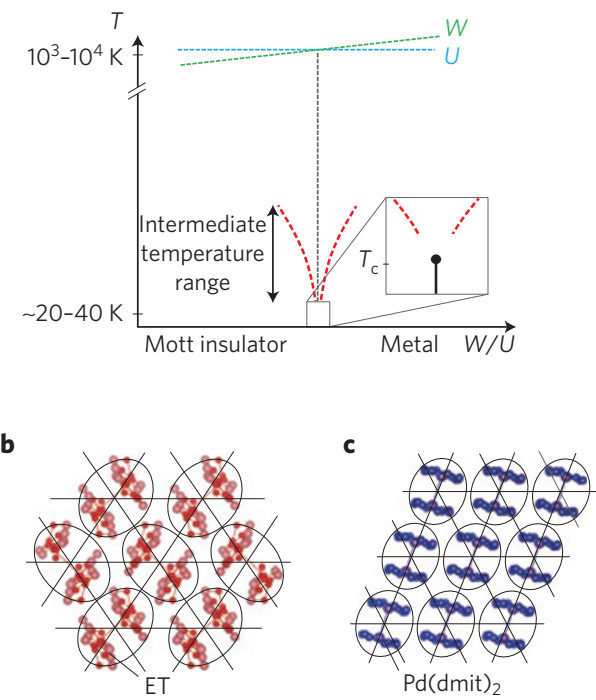
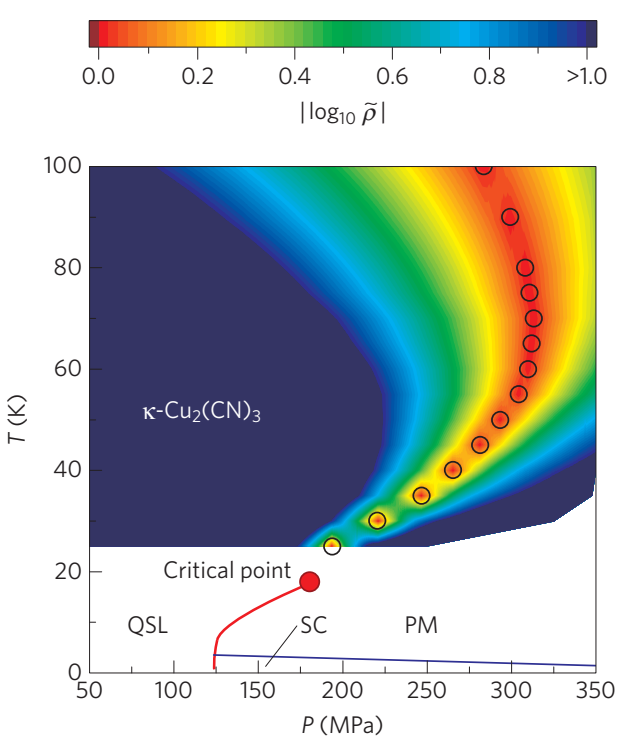

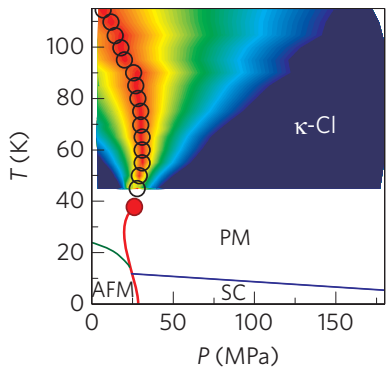

f

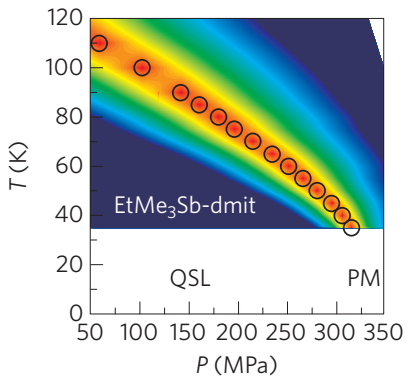

Figure 1 | The structure and phase diagram of organic Mott systems. a, Schematic phase diagram of the Mott transition. $\mathbf{b}, \mathbf{c}$, Structure of a conducting

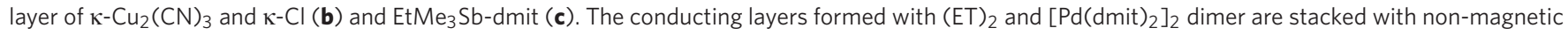
insulating layers, alternately. One (ET) $2\left(\left[\mathrm{Pd}(\mathrm{dmit})_{2}\right]_{2}\right)$ dimer has one hole (electron) and corresponds to a lattice site of an anisotropic triangular lattice. Strong on-site Coulomb repulsion and a half-filled band make the system a Mott insulator. $\mathbf{d}-\mathbf{f}$, Pressure-temperature phase diagram of $\kappa_{-}-\mathrm{Cu}_{2}(\mathrm{CN})_{3}$ (d), $\kappa-\mathrm{Cl}(\mathbf{e})$ and $\mathrm{EtMe}_{3} \mathrm{Sb}$-dmit (f). The red line represents the first-order Mott transition line terminating at a critical end point. The open circles indicate the metal-insulator crossover pressure $P_{c}(T)$ defined in the text. The colour represents the magnitude of $\left|\log _{10} \tilde{\rho}\right|$, where $\tilde{\rho}$ is the normalized resistivity defined in the text. The lowest temperature experimentally accessible for EtMe 3 Sb-dmit is limited to $35 \mathrm{~K}$, below which the He medium is solidified at pressures of interest for $\mathrm{EtMe}_{3} \mathrm{Sb}$-dmit.

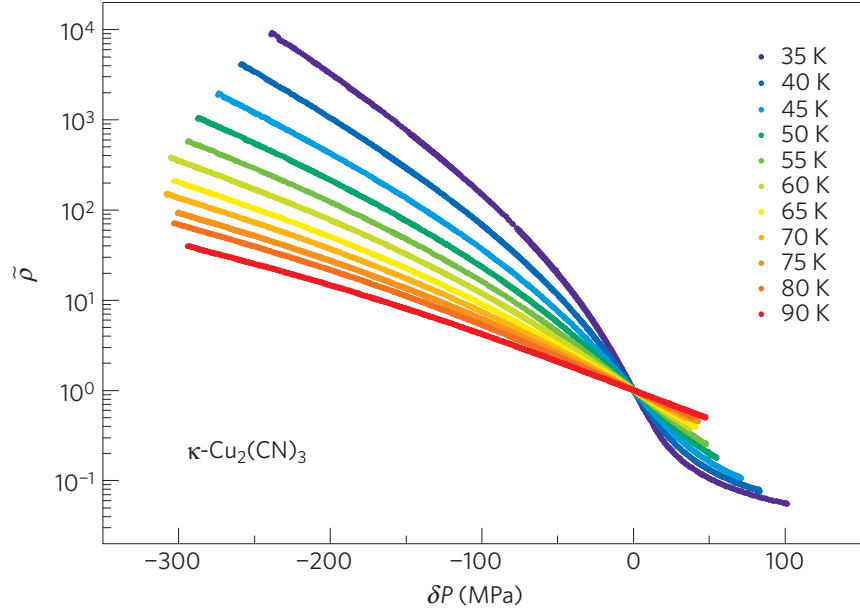

Figure 2 | The normalized resistivity $\tilde{\rho}(\delta P, T)$ of $\kappa-\mathrm{Cu}_{2}(\mathrm{CN})_{3}$ measured from the metal-insulator crossover line against $\delta P=P-P_{c}(T)$.

parameter controlling the quantum fluctuations, $g_{c}$ is the critical value of $g, v$ is the critical exponent of the correlation length, and $z$ is the dynamical exponent. At finite temperatures, another timescale, $L_{T} \propto T^{-1}$ appears because of a thermal effect; $L_{T}$ corresponds to the system size in the temporal direction, where $L_{T} \rightarrow \infty$ when $T \rightarrow 0$. At finite temperatures, finite-size scaling is available, such that the ratio of $L_{T}$ to $\tau$ determines the development of critical fluctuations. Consequently, the singular parts of physical quantities are governed by one scaling parameter, $\tau / L_{T} \propto T /\left|g-g_{\mathrm{c}}\right|^{z \nu}$. In the present study, considering the bow-shaped Widom line, it is natural to adopt $\delta P=P-P_{\mathrm{c}}(T)$ as the parameter controlling the quantum fluctuations instead of $P-P_{c}(T \rightarrow 0)$. Accordingly, if $\tilde{\rho}(\delta P, T)$ can be expressed as $\tilde{\rho}(\delta P, T)=F\left[T /|c \delta P|^{z \nu}\right]$, where $c$ is an arbitrary constant, and $F(y)$ is a scaling function of the scaling variable
$y=T /|c \delta P|^{z v}$, such that $F(y) \rightarrow 1$ when $y \rightarrow \infty$, we can conclude that the system is in the quantum-critical regime.

Turning to the experimental data for $\kappa-\mathrm{Cu}_{2}(\mathrm{CN})_{3}$, all data for $\tilde{\rho}(\delta P, T)$ in a wide temperature range $(35 \mathrm{~K} \leq T \leq 90 \mathrm{~K})$ collapse onto bifurcating scaling curves against $T / T_{0} \equiv T /|c \delta P|^{z v}$, with a critical exponent of $z v=0.62 \pm 0.02$, as shown in Fig. 3a. The scaling is fulfilled over several orders of magnitude. For $\kappa-\mathrm{Cl}$ and $\mathrm{EtMe}_{3} \mathrm{Sb}$-dmit, similar scaling is observed for $75 \mathrm{~K} \leq T \leq 115 \mathrm{~K}$ with $z v=0.49 \pm 0.01$ (Fig. 3b) and for $35 \mathrm{~K} \leq T \leq 90 \mathrm{~K}$ with $z v=0.68 \pm 0.04$ (Fig. 3c), respectively (Supplementary Information). The scaling curves for the three systems nearly coincide with each other (Supplementary Information). It is remarkable that these three systems with different ground states show common scaling behaviour; this implies that the present Mott quantum criticality in the intermediate temperatures comes from the high-energy itinerant-localized competition, no matter which ground state the system falls into at low temperatures. These scaling curves clearly reveal the perfect 'mirror symmetry' of $\tilde{\rho}(\delta P, T)$ between the insulating $(\tilde{\rho}>1)$ and metallic $(\tilde{\rho}<1)$ branches at $T / T_{0} \gtrsim 1$. The mirror symmetry indicates that the scaling function $F(y)$ exhibits duality at $y \gtrsim 1$ between the metallic $(\delta P>0)$ and insulating $(\delta P<0)$ sides as $F_{\text {metal }}(y)=1 / F_{\text {insulator }}(y)$. It is noticeable that this duality of scaling is also observed in a metalinsulator transition in the two-dimensional electron gas system of a metal-oxide-semiconductor field-effect $\operatorname{transistor~}^{23}$ (MOSFET), where the electron correlation is argued to play a key role in the metal-insulator transition, possibly a Wigner crystallization ${ }^{24}$.

The form of scaling function is explained by a typical quantumcritical regime. At $T / T_{0} \gg 1$, the system does not 'know' whether it resides in the metallic or insulating side because of the large quantum-critical fluctuations. As $T / T_{0}$ decreases, the system gradually 'notices' the regime to which it belongs. The $\tilde{\rho}(\delta P, T)$ of the $\kappa-\mathrm{Cu}_{2}(\mathrm{CN})_{3}$ versus $T / T_{0}$ plot in Fig. $3 \mathrm{a}$ is shown using logarithmic scales in Fig. 4, which illustrates that for further decreases in $T / T_{0}$, the metallic branch deviates from the quantum-critical behaviour 


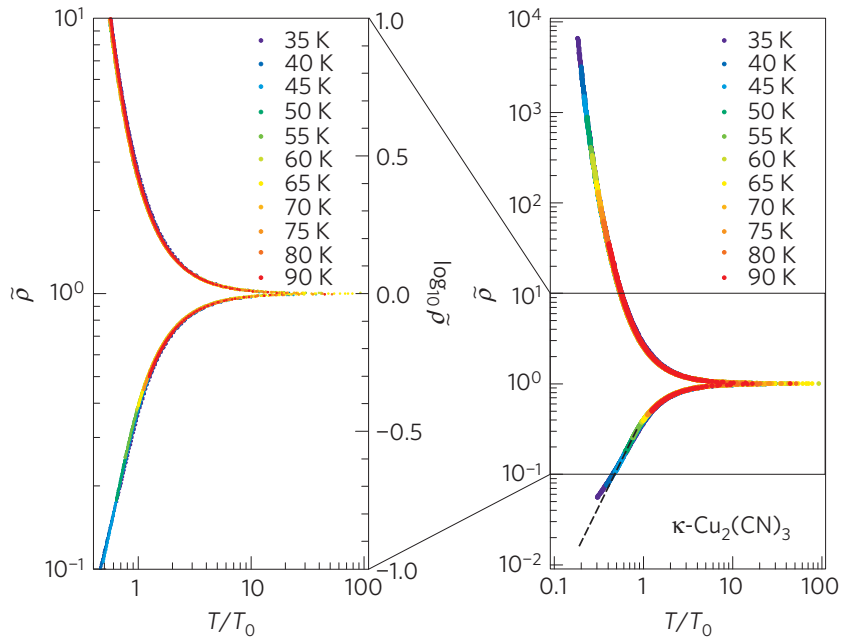

b

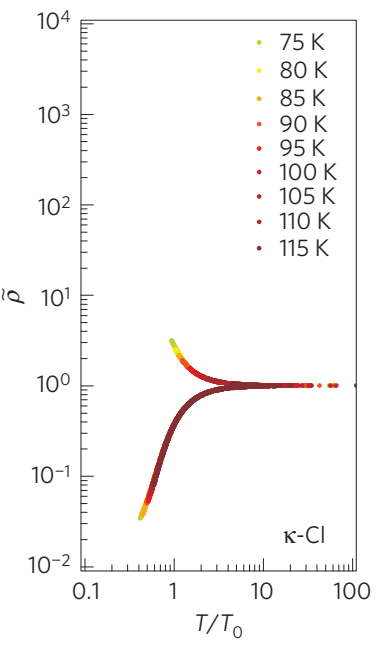

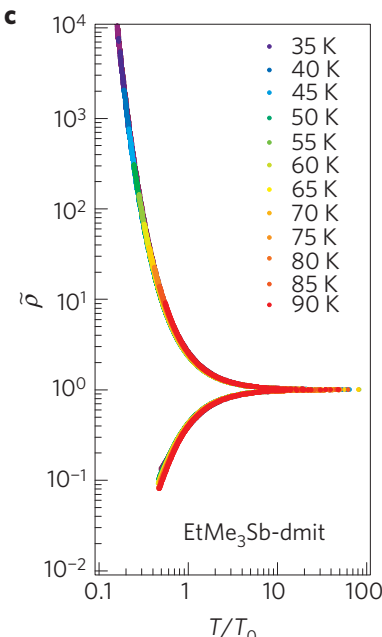

Figure 3 | Scaling analysis. a-c, The scaling plot of the normalized resistivity $\tilde{\rho}(\delta P, T)$ versus $T / T_{0}=T /|c \delta P|^{z v}$ with the present values, $z \nu=0.62$ and $c=20.9$ for $\kappa-\mathrm{Cu}_{2}(\mathrm{CN})_{3}(\mathbf{a}), z v=0.49$ and $c=289$ for $\mathrm{\kappa}-\mathrm{Cl}(\mathbf{b})$, and $z v=0.68$ and $c=13.1$ for EtMe $3 \mathrm{Sb}$-dmit (c). The upper and lower curves are the insulating and metallic branches, respectively. The dashed line in $\mathbf{a}$ indicates that $\tilde{\rho} \propto\left(T / T_{0}\right)^{2}$. The reason why the $\kappa-\mathrm{Cl}$ data are not extended in the insulating branch is that the Mott critical pressure of $\kappa-\mathrm{Cl}$ is so low that the insulating region would be extended at negative pressures experimentally inaccessible.

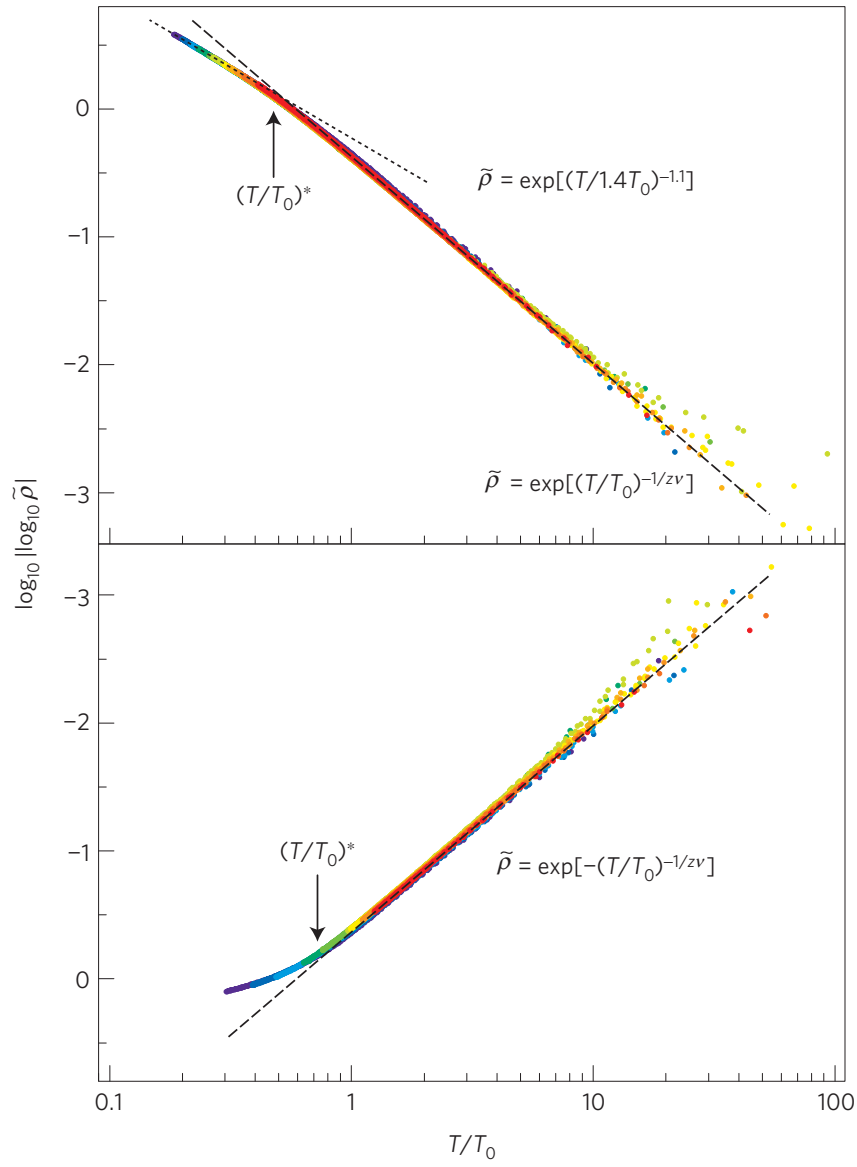

Figure 4 | Crossover behaviour of the scaling curve. Plot of $\log _{10}\left|\log _{10} \tilde{\rho}\right|$ versus $T / T_{0}$ for $\kappa-\mathrm{Cu}_{2}(\mathrm{CN})_{3}$. The upper and lower panels are the insulating and metallic branches, respectively. The dashed and dotted lines correspond to quantum-critical behaviour $\tilde{\rho}=\exp \left[ \pm\left(T / T_{0}\right)^{-1 / z v}\right]$ ( $z v=0.62,+$ : insulating branch, $-:$ metallic branch) and nearly activation-type behaviour $\tilde{\rho}=\exp \left[\left(T / 1.4 T_{0}\right)^{-1.1}\right]$, respectively. The arrows indicate the characteristic value of $T / T_{0}$, represented as $\left(T / T_{0}\right)^{*}$, for the entrance to the low-temperature regimes of the gapped Mott insulator or the Fermi liquid, determined by eye. following $\tilde{\rho}=\exp \left[-\left(T / T_{0}\right)^{-1 / z v}\right]$ towards the Fermi-liquid $T^{2}$ behaviour (Fig. 3a), followed by saturation to the residual resistivity, and the insulating branch also deviates from quantum-critical behaviour $\tilde{\rho}(\delta P, T)=\exp \left[\left(T / T_{0}\right)^{-1 / z \nu}\right]$ towards near-activation behaviour fitted by the form $\tilde{\rho}(\delta P, T)=\exp \left[\left(T / 1.4 T_{0}\right)^{-1.1}\right]$ with a gap of the order of $T_{0}$. Similar behaviour is also observed for the other two compounds (see Supplementary Fig. 5). These behaviours at low $T / T_{0}$ indicate the entrance of the high-temperature quantumcritical state into the low-temperature regimes of the Fermi liquid or the gapped Mott insulator. The $T_{0}$ values for the three compounds, which characterize quantum-critical regions, are shown in Supplementary Fig. 4.

The normalized resistivity $\tilde{\rho}(P, T)$ is represented by a range of colour in Fig. 1d-f, to visualize the quantum-critical region (the red, yellow and green regions), where the mirror symmetry in Fig. 3a-c is near-perfect. A depiction of $\tilde{\rho}$ in the $\delta P-T$ plane instead of the $P-T$ plane highlights the mirror symmetry of the quantum-critical region, as shown in Supplementary Fig. 6.

The scaling features of the present data surprisingly coincide with those of recent DMFT calculations ${ }^{4,13}$. The most remarkable point is that both the experimental and theoretical scaling curves have the same functional form, with the mirror symmetry. Moreover, the critical exponent $z v=0.57$ in DMFT (ref. 4) is close to the present values $z \nu=0.62$ for $\kappa-\mathrm{Cu}_{2}(\mathrm{CN})_{3}, 0.49$ for $\kappa-\mathrm{Cl}$, and 0.68 for $\mathrm{EtMe}_{3} \mathrm{Sb}$-dmit. It is also noticeable that these experimental values are comparable to $z v=0.67$, a value expected for the genuine quantum Mott transition ${ }^{25}$. Although there seem to be appreciable differences between the $z v$ values $(0.62 \pm 0.02,0.49 \pm 0.02$ and 0.68 \pm 0.04 ), we note that they are in a window of $0.6 \pm 0.1$ and that the differences between the present $z v$ values are much smaller than the possible range of $z v$ values for quantum metal-insulator transitions; for example, a value of $z v=2(z=4, v=1 / 2)$ is proposed theoretically for the marginal quantum Mott transition in ref. 6 and a value of $z v=1.6(z=1, v=1.6)$ is deduced experimentally in a Si-MOSFET in ref. 26. Thus, the present results suggest a possible universality class with a $z v$ value around 0.6. However, we do not exclude a possibility that the small distribution in $z v$ values is a signature of an intriguing case with non-universal critical exponents such as the Berezinskii-Kosterlitz-Thouless transition ${ }^{27}$, where the universality can be characterized by lines of fixed points in renormalization group theory, and the variation of $z v$ comes from the microscopic parameters of material such as lattice geometry. In the DMFT work, 
the temperature range in which the scaling is valid is approximately $2 T_{\mathrm{c}}<T<0.12 t$, where $t$ is the nearest-neighbour transfer integral in the Hubbard model. Both the lower and upper limits nearly coincide with the present results. Furthermore, the bow-shaped crossover line and the fan-shaped quantum-critical region are common to both the present and the DMFT works. As DMFT can treat only fluctuations independent of wavenumber $k$, the present agreement may indicate that the fluctuations of Mott quantum criticality, where electrons are neither particle-like nor wave-like objects, can be of the $k$-independent local nature.

In general, quantum-critical behaviour springs from a quantum phase transition associated with symmetry breaking. However, the Mott quantum criticality in question is associated with the charge delocalization transition without symmetry breaking and has been actually demonstrated here to occur irrespectively of the presence/absence of symmetry breaking in spin degrees freedom. Thus, the present quantum criticality is beyond the conventional symmetry-breaking framework. Unconventional quantum criticality has also been discussed for heavy-electron systems in light of the Kondo breakdown, where the reconstruction of Fermi surfaces or an orbital-selective Mott transition due to itinerant-localized competition of $f$ electrons supposedly causes unconventional quantum criticality ${ }^{28,29}$. Moreover, theoretical investigations of the holographic duality of current interest have proposed that strange metals in heavy electrons and cuprates are in yet-unspecified quantum-critical regimes ${ }^{30,31}$. It is likely that correlated quantum systems of organics, heavy electrons and cuprates carry a new class of quantum criticality that originates from the itinerant-localized competition rather than symmetry breaking.

\section{Methods}

Single crystals of $\kappa-(\mathrm{ET})_{2} \mathrm{Cu}_{2}(\mathrm{CN})_{3}$ and $\kappa-(\mathrm{ET})_{2} \mathrm{Cu}\left[\mathrm{N}(\mathrm{CN})_{2}\right] \mathrm{Cl}$ were grown by conventional electrochemical oxidation. Single crystals of $\mathrm{EtMe}_{3} \mathrm{Sb}\left[\mathrm{Pd}(\mathrm{dmit})_{2}\right]_{2}$ were obtained by air oxidation of $\left(\mathrm{EtMe}_{3} \mathrm{Sb}\right)_{2}\left[\mathrm{Pd}(\mathrm{dmit})_{2}\right]$ in acetone containing acetic acid ${ }^{32}$. The typical sample sizes are $\sim 0.3 \mathrm{~mm} \times 0.3 \mathrm{~mm} \times 0.006 \mathrm{~mm}$ for $\kappa-(\mathrm{ET})_{2} \mathrm{Cu}_{2}(\mathrm{CN})_{3}, \sim 1.0 \mathrm{~mm} \times 0.5 \mathrm{~mm} \times 0.2 \mathrm{~mm}$ for $\kappa-(\mathrm{ET})_{2} \mathrm{Cu}\left[\mathrm{N}(\mathrm{CN})_{2}\right] \mathrm{Cl}$, and $\sim 0.8 \mathrm{~mm} \times 0.9 \mathrm{~mm} \times 0.05 \mathrm{~mm}$ for $\mathrm{EtMe}_{3} \mathrm{Sb}\left[\mathrm{Pd}(\mathrm{dmit})_{2}\right]_{2}$. The in-plane electrical resistivity was measured using the standard d.c. four-probe method under an isothermal pressure sweep (descending processes), using helium gas as the pressure medium. To examine the sample dependence, measurements were performed for two samples of the same compound simultaneously in the same run. The reproducibility of the scaling behaviour was basically ensured for each compound. Gold wires of $25 \mu \mathrm{m}$ in diameter were glued on the crystal faces with carbon paste as electrodes. Throughout the experiments, we confirmed that the resistivity was independent of the applied current.

Received 11 March 2014; accepted 23 December 2014; published online 9 February 2015

\section{References}

1. Sachdev, S. Quantum Phase Transitions (Cambridge Univ. Press, 2011).

2. Sondhi, S. L., Girvin, S. M., Carini, J. P. \& Shahar, D. Continuous quantum phase transitions. Rev. Mod. Phys. 69, 315-333 (1997)

3. Mott, N. F. Metal-Insulator Transitions (Taylor \& Francis, 1990).

4. Terletska, H., Vučičević, J., Tanasković, D. \& Dobrosavljević, V. Quantum critical transport near the Mott transition. Phys. Rev. Lett. 107, 026401 (2011).

5. Senthil, T. Theory of a continuous Mott transition in two dimensions. Phys. Rev. B 78, 045109 (2008).

6. Misawa, T. \& Imada, M. Quantum criticality around metal-insulator transitions of strongly correlated electron systems. Phys. Rev. B 75, 115121 (2007).

7. McWhan, D., Menth, A., Remeika, J., Brinkman, W. \& Rice, T. Metal-insulator transitions in pure and doped $\mathrm{V}_{2} \mathrm{O}_{3}$. Phys. Rev. B 7, 1920-1931 (1973).

8. Yao, X., Honig, J., Hogan, T., Kannewurf, C. \& Spałek, J. Electrical properties of $\mathrm{NiS}_{2-x} \mathrm{Se}_{x}$ single crystals: From Mott insulator to paramagnetic metal. Phys. Rev. B 54, 17469-17475 (1996).

9. Lefebvre, S. et al. Mott transition, antiferromagnetism, and unconventional superconductivity in layered organic superconductors. Phys. Rev. Lett. 85, 5420-5423 (2000).

10. Limelette, $\mathrm{P}$. et al. Mott transition and transport crossovers in the organic compound $\kappa-(\mathrm{BEDT}-\mathrm{TTF})_{2} \mathrm{Cu}\left[\mathrm{N}(\mathrm{CN})_{2}\right] \mathrm{Cl}$. Phys. Rev. Lett. 91, 016401 (2003).
11. Kagawa, F., Miyagawa, K. \& Kanoda, K. Unconventional critical behaviour in a quasi-two-dimensional organic conductor. Nature 436, 534-537 (2005).

12. Georges, A., Kotliar, G., Krauth, W. \& Rozenberg, M. J. Dynamical mean-field theory of strongly correlated fermion systems and the limit of infinite dimensions. Rev. Mod. Phys. 68, 13-125 (1996).

13. Vučičević, J., Terletska, H., Tanasković, D. \& Dobrosavljević, V. Finite-temperature crossover and the quantum Widom line near the Mott transition. Phys. Rev. B 88, 075143 (2013).

14. Shimizu, Y., Miyagawa, K., Kanoda, K., Maesato, M. \& Saito, G. Spin liquid state in an organic Mott insulator with a triangular lattice. Phys. Rev. Lett. 91, 107001 (2003)

15. Itou, T., Oyamada, A., Maegawa, S., Tamura, M. \& Kato, R. Quantum spin liquid in the spin-1/2 triangular antiferromagnet $\mathrm{EtMe}_{3} \mathrm{Sb}\left[\mathrm{Pd}(\mathrm{dmit})_{2}\right]_{2}$. Phys. Rev. B 77, 104413 (2008).

16. Kanoda, K. \& Kato, R. Mott physics in organic conductors with triangular lattices. Annu. Rev. Condens. Matter Phys. 2, 167-188 (2011).

17. Kato, R. Development of $\pi$-electron systems based on $\left[\mathrm{M}(\mathrm{dmit})_{2}\right](\mathrm{M}=\mathrm{Ni}$ and Pd; dmit: 1,3-dithiole-2-thione-4,5-dithiolate) anion radicals. Bull. Chem. Soc. Jpn 87, 355-374 (2014).

18. Powell, B. J. \& McKenzie, R. H. Quantum frustration in organic Mott insulators: From spin liquids to unconventional superconductors. Rep. Prog. Phys. 74, 056501 (2011).

19. Komatsu, T., Matsukawa, N., Inoue, T. \& Saito, G. Realization of superconductivity at ambient pressure by band-filling control in к-(BEDT-TTF $)_{2} \mathrm{Cu}_{2}(\mathrm{CN})_{3}$. J. Phys. Soc. Jpn 65, 1340-1354 (1996).

20. Kurosaki, Y., Shimizu, Y., Miyagawa, K., Kanoda, K. \& Saito, G. Mott transition from a spin liquid to a Fermi liquid in the spin-frustrated organic conductor К-(ET) ${ }_{2} \mathrm{Cu}_{2}(\mathrm{CN})_{3}$. Phys. Rev. Lett. 95, 177001 (2005).

21. Kato, R., Tajima, A., Nakao, A., Tajima, N., Tamura, M. in Multifunctional Conducting Molecular Materials (eds Saito, G. et al.) 32-38 (Royal Society of Chemistry, 2007).

22. Kobashi, K. Transport Properties Near the Mott Transition in the Quasi-Two-Dimensional Organic Conductor $\kappa-(E T)_{2} X$ Thesis, Univ. Tokyo (2007)

23. Simonian, D., Kravchenko, S. V. \& Sarachik, M. P. Reflection symmetry at a $B=0$ metal-insulator transition in two dimensions. Phys. Rev. B 55, R13421-R13423 (1997).

24. Amaricci, A. et al. Extended Hubbard model: Charge ordering and Wigner-Mott transition. Phys. Rev. B 82, 155102 (2010).

25. Witczak-Krempa, W., Ghaemi, P., Senthil, T. \& Kim, Y. B. Universal transport near a quantum critical Mott transition in two dimensions. Phys. Rev. B 86, 245102 (2012)

26. Kravchenko, S. et al. Scaling of an anomalous metal-insulator transition in a two-dimensional system in silicon at $B=0$. Phys. Rev. B 51, 7038-7045 (1995).

27. Kosterlitz, J. M. The critical properties of the two-dimensional xy model. J. Phys. C 7, 1046-1060 (1974).

28. Si, Q., Rabello, S., Ingersent, K. \& Smith, J. L. Locally critical quantum phase transitions in strongly correlated metals. Nature 413, 804-808 (2001)

29. Senthil, T., Sachdev, S. \& Vojta, M. Quantum phase transitions out of the heavy Fermi liquid. Physica B 359-361, 9-16 (2005).

30. Sachdev, S. Holographic metals and the fractionalized Fermi liquid. Phys. Rev. Lett. 105, 151602 (2010).

31. Davison, R. A., Schalm, K. \& Zaanen, J. Holographic duality and the resistivity of strange metals. Phys. Rev. B 89, 245116 (2014)

32. Kato, R. \& Hengbo, C. Cation dependence of crystal structure and band parameters in a series of molecular conductors, $\beta^{\prime}-($ Cation $)\left[\operatorname{Pd}(\mathrm{dmit})_{2}\right]_{2}$ (dmit = 1,3-dithiole-2-thione-4,5-dithiolate). Crystals 2, 861-874 (2012).

\section{Acknowledgements}

We would like to thank V. Dobrosavljević, N. Nagaosa, H. Oike and T. Itou for fruitfu discussions. This work was supported in part by JSPS KAKENHI under Grant Nos 20110002, 25220709 and 24654101, and the US National Science Foundation under Grant No. PHYS-1066293 and the hospitality of the Aspen Center for Physics.

\section{Author contributions}

T.F. and K.K. designed the experiments. T.F. performed the experiments and analysed the data. T.F. and K.K. interpreted the data. K.M., H.T. and R.K. grew the single crystals for the study. T.F. wrote the manuscript with the assistance of K.M. and K.K. K.K. headed this project.

\section{Additional information}

Supplementary information is available in the online version of the paper. Reprints and permissions information is available online at www.nature.com/reprints.

Correspondence and requests for materials should be addressed to T.F. or K.K.

\section{Competing financial interests}

The authors declare no competing financial interests. 